\title{
Compreender a Sintomatologia Depressiva após a Cirurgia Bariátrica: o Papel do Peso, da Alimentação e da Imagem Corporal
}

\author{
Understanding Depressive Symptoms after Bariatric Surgery: the Role of \\ Weight, Eating and Body Image
}

\author{
Paula SOUSA ${ }^{1}$, Ana Pinto BASTOS ${ }^{1}$, Carla VENÂNCIO ${ }^{2}$, Ana Rita VAZ ${ }^{1}$, Isabel BRANDÃO ${ }^{3}$, José Maia da COSTA ${ }^{4}$, \\ Paulo MACHADO ${ }^{1}$, Eva CONCEIÇÃO ${ }^{1}$ \\ Acta Med Port 2014 Jul-Aug;27(4):450-457
}

\section{RESUMO}

Introdução: A sintomatologia depressiva tem-se revelado prevalente após a cirurgia bariátrica. Este estudo teve como objetivo compreender o papel do peso, alimentação e imagem corporal na sintomatologia depressiva após realização da cirurgia bariátrica.

Material e Métodos: Segue um design transversal e possui uma amostra pós-cirúrgica de 52 indivíduos, com um tempo de follow-up entre os 22 e os 132 meses. A avaliação foi realizada através de duas entrevistas clínicas semiestruturadas, incluindo a perturbação de comportamento alimentar (Eating Disorder Examination), e de três instrumentos de autorrelato: Outcome Questionnaire 45 - comprometimento psicológico geral, Beck Depression Inventory - sintomatologia depressiva e Body Shape Questionnaire - imagem corporal. Resultados: Os resultados mostram que a sintomatologia depressiva está associada à ingestão alimentar compulsiva, à preocupação com a imagem corporal e à percentagem do índice de massa corporal ganho. Segundo os resultados da análise de regressão linear múltipla que incluiu estas variáveis, a percentagem de índice de massa corporal ganho após cirurgia, a ingestão alimentar compulsiva e a preocupação com a imagem corporal são os factores que explicam $50 \%$ da variância da sintomatologia depressiva após a cirurgia, sendo a preocupação com a imagem corporal mais significativo, na medida em que uma maior insatisfação com a imagem corporal está associada a mais sintomas depressivos.

Discussão: Os resultados deste estudo mostraram que um subgrupo de doentes apresenta um aumento de peso significativo após a cirurgia bariátrica que está associado a episódios de ingestão alimentar compulsiva, a preocupações com a imagem corporal e a sintomatologia depressiva.

Conclusão: Estes resultados enfatizam a importância de considerar estas preocupações com a imagem corporal na avaliação psicológica da pessoa que fez cirurgia bariátrica de modo a otimizar o seu funcionamento psíquico e adesão ao tratamento.

Palavras-chave: Depressão; Cuidados Pré-Operatórios; Escalas de Graduação Psiquiátrica; Obesidade Mórbida; Imagem Corporal.

\section{ABSTRACT}

Introduction: Depressive symptoms have been reported as prevalent after bariatric surgery. This study aims to analyze the role of weight, eating behaviors and body image in depressive symptomatology in bariatric surgery patients assessed post-operatively.

Material and Methods: This is a cross-sectional study including 52 bariatric surgery patients assessed post-operatively with a follow-up time ranging from 22 to 132 months. Psychological assessment included a clinical interview (Eating Disorder Examination) to assess eating disorders psychopathology, and three self-report measures: Outcome Questionnaire 45 - general distress; Beck Depression Inventory - depressive symptoms; and Body Shape Questionnaire - body image.

Results: Our data show that depressive symptoms after surgery are associated with loss of control over eating, increased concerns with body image, and body mass index regain. Multiple linear regressions was tested including these variables and showed that body mass index regain after surgery, loss of control over eating and concerns with body image significantly explained $50 \%$ of the variance of post-operative depressive symptoms, being the concern with body image the most significant variable: greater dissatisfaction with body image was associated with more depressive symptoms.

Discussion: The results of this study showed that a subgroup of patients presents a significant weight gain after bariatric surgery, which is associated with episodes of loss of control over eating, concerns with body image and depressive symptoms.

Conclusion: These results stress the relevance of body image concerns after surgery and the importance of clinically addressing these issues to optimize psychological functioning after bariatric surgery.

Keywords: Depression; Preoperative Care; Psychiatric Status Rating Scales; Obesity, Morbid; Body Image; Bariatric Surgery.

\section{INTRODUÇÃo}

A obesidade, reconhecida atualmente como a doença do século XXI, é um problema de saúde pública em crescimento. ${ }^{1,2} \mathrm{O}$ número de obesos mais do que duplicou mundialmente desde 1980 e em 2008 cerca de 1,4 milhões da população adulta Mundial tinha excesso de peso. ${ }^{3}$ Entre Janeiro de 2003 e Janeiro de 2005, em Portugal, mais de metade da população portuguesa tinha excesso de peso ou era obesa $\left(39,9 \%\right.$ e $14,2 \%$, respetivamente). ${ }^{4}$
A depressão tem sido salientada por clínicos e investigadores no tratamento da obesidade e estudos recentes mostram a existência de uma relação bidirecional entre depressão e obesidade. ${ }^{5}$ Fatores psicológicos e psicossociais como estratégias de coping deficitárias e uma baixa auto-estima, também presentes na depressão, parecem estar associados a comportamentos alimentares disfuncionais e à obesidade. ${ }^{6}$ Um estudo que analisou a sintomatologia

1. Escola de Psicologia. Centro de Investigação em Psicologia. Universidade do Minho. Braga. Portugal.

2. Unidade de Bariatria. Clínica Dr. António Sérgio. Instituto CUF Porto. Senhora da Hora. Portugal.

3. Departamento de Psicologia. Faculdade de Medicina. Universidade do Porto. Hospital de São João. Porto. Portugal.

4. Serviço de Cirurgia. Hospital de Braga. Braga. Portugal.

Recebido: 14 de Outubro de 2013 - Aceite: 19 de Maio de 2014 | Copyright @ Ordem dos Médicos 2014 
depressiva numa população com obesidade e excesso de peso, comparando indivíduos com depressão e sem depressão, revela que o grupo de indivíduos com depressão apresenta mais comportamentos de ingestão alimentar compulsiva que os indivíduos sem depressão. ${ }^{7}$ Este estudo demonstra ainda que existe uma correlação significativa entre o índice de massa corporal (IMC) e sintomas depressivos (BDI), assim como o aumento de sintomas depressivos quando o IMC aumenta. ${ }^{7}$

A depressão na obesidade também está associada à preocupação com a imagem corporal, sendo a insatisfação com a imagem corporal significativamente maior na população de obesos em comparação com indivíduos de peso normal e associada a uma maior incidência de depres-

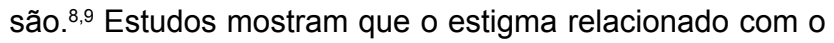
peso nas pessoas obesas conduz a uma menor satisfação com o seu corpo e forma, assim como a uma autoestima diminuída, o que poderá estar associado ao aparecimento da sintomatologia depressiva. ${ }^{5,10}$ Adicionalmente, e devido às situações de discriminação associadas ao peso, os indivíduos obesos que possuem uma visão mais negativa da imagem corporal possuem um maior risco de desenvolver depressão. ${ }^{11}$

Apesar dos bons resultados dos tratamentos convencionais para a obesidade como programas de modificação comportamental ou dietas alimentares, estes não possuem resultados satisfatórios para a obesidade severa, sendo a cirurgia bariátrica considerada o tratamento de eleição uma vez que resulta numa perda de peso significativa com manutenção dos resultados a longo prazo, melhorias nas comorbilidades (i.e. diabetes, hipertensão), diminuição da psicopatologia, normalização dos padrões alimentares disfuncionais e melhorias na qualidade de vida e relações interpessoais. ${ }^{12-19}$

Contudo, os resultados da cirurgia bariátrica nem sempre são satisfatórios e uniformes. Alguns doentes não experienciam perdas de peso significativas ou têm mesmo um ganho de peso após a perda inicial. ${ }^{13}$ Estudos revelam que a maior quantidade de peso perdido ocorre especialmente logo após a cirurgia e ao longo dos dois primeiros anos, mantendo-se ou mesmo aumentando após esse período. ${ }^{19}$ Estas alterações de peso devem-se a inúmeras causas, incluindo fatores não-cirúrgicos que parecem influenciar os resultados da cirurgia bariátrica. ${ }^{13,19,20}$

O interesse pelo estudo das variáveis psicológicas associadas à cirurgia bariátrica tem aumentado significativamente nos últimos anos. Parecem existir evidências claras acerca da influência destas variáveis na vida das pessoas obesas e nos resultados dos tratamentos para a obesidade influenciando a perda de peso, a adesão ao pós-operatório e o cumprimento das prescrições médicas e da dieta alimentar. ${ }^{13-16,21-24}$

Uma das variáveis psicológicas que tem merecido particular interesse é a depressão. Estudos mostram que a existência de uma perturbação depressiva pré ou pós-cirurgia influencia consideravelmente a recuperação e os resultados do tratamento cirúrgico, estando associada a preocupações com a aparência, baixa autoestima, episódios de ingestão alimentar compulsiva e com alterações de peso. ${ }^{16,24,25}$

Após a cirurgia os sintomas depressivos pré-cirurgia tendem a diminuir, ${ }^{15,16}$ mas estas melhorias parecem limitadas ao primeiro ano após a cirurgia, ${ }^{13,24}$ uma vez que 24 meses após a cirurgia parece existir uma continuidade ou emergência de sintomatologia depressiva. Este é considerado um momento de mudança ao nível da psicopatologia com dissipação das melhorias psicológicas obtidas ao longo do primeiro ano após a cirurgia. ${ }^{15,25,26}$

A literatura é consistente quanto às variáveis associadas à depressão na obesidade, demonstrando uma relação entre a depressão, o comportamento alimentar, o aumento de peso e a imagem corporal. ${ }^{5-11}$ Contudo, após a cirurgia bariátrica, que resulta numa perda de peso muito acentuada, os estudos falham em perceber quais as variáveis associadas à sintomatologia depressiva. É consistente que a depressão assume um papel importante na perda de peso e na presença de comportamento alimentar disfuncional após a cirurgia, diminuindo logo após a cirurgia mas reaparecendo passados dois anos..$^{8,18,25}$ Após a cirurgia, muitos dos indivíduos com perda significativa de peso não apresentam melhorias ao nível dos sintomas depressivos. ${ }^{19}$ É importante perceber que outras variáveis podem estar associadas à sintomatologia depressiva após a cirurgia, para que se possam identificar soluções que conduzam a um menor comprometimento psicológico e a resultados mais satisfatórios no tratamento da obesidade.

Este estudo tem como objetivo compreender a associação da perda de peso, comportamento alimentar e imagem corporal à sintomatologia depressiva em doentes com um follow-up cirúrgico entre os 22 e 132 meses.

\section{MATERIAL E MÉTODOS}

Foram recolhidos dados de um estudo longitudinal ainda em curso sobre os preditores de sucesso na cirurgia bariátrica, levado a cabo pelo Grupo de Estudos das Perturbações Alimentares da Escola de Psicologia, Universidade do Minho. Para o presente trabalho, foram selecionados 52 indivíduos que preenchiam os critérios de inclusão estabelecidos: ter realizado cirurgia há mais de dois anos (aproximadamente) e ter avaliação de sintomatologia depressiva. Foram excluídos para este estudo indivíduos com perturbações depressivas major, de ingestão alimentar compulsiva aguda e com bulimia nervosa. Esta avaliação foi feita pelo médico psiquiatra no contexto da avaliação do paciente para parecer positivo para este tratamento, tendo parecer negativo doentes que apresentassem perturbações de humor ou perturbações do comportamento alimentar, nomeadamente doentes que apresentem perturbações depressivas major, bulimia nervosa ou de ingestão alimentar compulsiva. A avaliação de sujeitos pós-cirurgia seguiu um design transversal. A seleção do tempo de follow-up após a cirurgia entre os 22 e os 132 meses considerou a premissa de que é após este período de tempo que a sintomatologia depressiva tende a emergir ou a reemergir e o facto 
da perda de peso já estar mais estabilizada a partir desse momento. ${ }^{19,24}$

Os doentes foram recrutados em três hospitais do norte de Portugal: 16 do Hospital de Braga, 16 do Hospital de S. João e 20 da Clínica Dr. António Sérgio - Unidade de Bariatria do Instituto CUF Porto. Como parte do protocolo de avaliação foram usadas duas entrevistas semiestruturadas e um conjunto de questionários de autorrelato (OQ45, $B S Q, B D I)$. As entrevistas clínicas foram realizadas por psicólogos treinados na avaliação e tratamento das perturbações do comportamento alimentar.

Neste estudo, a variável ingestão alimentar compulsiva compreende os episódios subjetivos e objetivos, uma vez que as duas condições demonstram características clínicas semelhantes, considerando-se a ingestão alimentar compulsiva independentemente da quantidade de comida que foi ingerida, tal como foi feito em estudos anteriores. ${ }^{27}$

O aumento de peso após a cirurgia foi considerado significativo quando este excedia $5 \%$ do peso corporal.

\section{Instrumentos}

Guião de entrevista clínica: inclui dados sociodemográficos e informações clínicas como IMC e história do peso.

Eating Disorder Examination (EDE) ${ }^{28}$ : Entrevista semiestruturada que avalia a frequência e severidade de sintomas de perturbação de comportamento alimentar nos últimos 28 dias. Foi validada para a população portuguesa ${ }^{29}$ e possui um conjunto de itens de diagnóstico que foram aplicados neste estudo e que permitem avaliar a presença de episódios subjetivos e/ou objetivos de ingestão alimentar compulsiva, com base nos critérios de diagnóstico do DSM-IV. Considera-se a perda de controlo alimentar quando o sujeito refere claramente ter perdido o controlo sobre o que comeu, ou sentiu-se 'levado ou compelido a comer', ou refere que não conseguiu parar de comer depois de ter começado revelando não ter sido capaz de travar o episódio, ou menciona que já nem tenta controlar a sua alimentação pois a ingestão é inevitável. Um episódio de ingestão alimentar é considerado subjetivo quando a quantidade de comida ingerida não é realmente excessiva, mas é considerada pelo indivíduo como tal, e objetivo quando compreende quantidades ingeridas realmente excessivas. A decisão se a quantidade de comida foi ou não excessiva é tomada pelo entrevistador, não exige o acordo do sujeito e deve ter em conta as circunstâncias em que ocorreu o episódio. O EDE tem sido vastamente utilizado na população portuguesa e alguns estudos referem-no como o método de eleição na avaliação da sintomatologia específica das perturbações do comportamento alimentar. ${ }^{30}$ As suas propriedades psicométricas são favoráveis, havendo estudos que confirmam a sua validade discriminativa, a sua fidelidade e consistência interna. ${ }^{31}$ Além disto, importa referir que o EDE foi o instrumento usado no maior e mais compreensivo estudo epidemiológico realizado em Portugal nas perturbações alimentares. ${ }^{32} \mathrm{Na}$ presente investigação o alfa de Cronbach para este instrumento foi de 0,92.
Beck Depression Inventory (BDI) ${ }^{33-35}$ : Permite avaliar a presença e gravidade subjetiva da sintomatologia depressiva, sendo composto por 21 itens com formato de resposta em escolha múltipla. Os sujeitos respondem em relação à última semana, cada questão é avaliada de 0 a 3 e o total resulta do somatório de todos os itens, variando entre 0 a 63 pontos. Valores mais elevados correspondem a mais sintomatologia depressiva. A versão portuguesa apresenta boas propriedades psicométricas e para a amostra em estudo apresentou um alfa de Cronbach de 0,85.

Outcome Questionnaire 45 - OQ45.2 ${ }^{36}$ : Questionário de autorrelato constituído por 45 itens, avalia o comprometimento psicológico geral e défice social. Os doentes respondem a uma escala de Likert de cinco pontos (nunca e sempre) e os itens geram três escalas (relações interpessoais, desempenho social e sintomas de comprometimento psicológico), além de uma escala global que resulta da soma das três escalas. Valores elevados na escala global indicam a presença de um conjunto de sintomas psicossociais e de desempenho social (escola, trabalho e qualidade de vida em geral). A versão portuguesa apresenta boas propriedades psicométricas (Machado PP, Fassnacht D, comunicação pessoal). Na nossa amostra foi utilizada a escala total com um alfa de Cronbach de 0,87.

Body Shape Questionnaire (BSQ) ${ }^{37}$ : Questionário de autorrelato constituído por 34 itens, que avalia preocupações com o corpo e a forma corporal tendo em conta as últimas quatro semanas. As respostas variam entre 0 e 6 , com as pontuações mais elevadas a corresponderem a uma maior insatisfação com a imagem corporal. Foi validado para a população portuguesa com boas propriedades psicométricas. ${ }^{38} \mathrm{Na}$ presente investigação, o alfa de Cronbach foi de 0,97 .

\section{Análises Estatísticas}

A percentagem de índice de massa corporal ganho após a cirurgia (\%IMCG) é representativa da percentagem de massa corporal que o indivíduo ganhou, relativamente ao IMC total perdido após a cirurgia e foi calculada da seguinte forma:

\%IMCG $=($ IMC_pós-cirurgia - IMC_mais baixo após a cirurgia) / (IMC_pré-cirurgia - IMC_mais baixo após a cirurgia) x 100. O IMC_pós-cirurgia representa o IMC no momento da avaliação pós-cirúrgica.

A percentagem de excesso de índice de massa corporal perdido após a cirurgia (percentagem EIMCP) representa a percentagem de excesso de massa corporal que o individuo perdeu após a cirurgia, tendo em conta o IMC ideal de 25 e foi calculada usando a fórmula:

$\%$ EIMCP = (IMC_pré-cirurgia - IMC_pós- cirurgia) $/($ IMC_ pré-cirurgia-25) x 100 .

Numa fase inicial foram investigadas correlações simples usando coeficientes de correlações de Pearson e Ponto-Bisserial com o objetivo de investigar quais as variáveis significativamente associadas à sintomatologia depressiva. Posteriormente as variáveis com significância estatística seriam incluídas em dois modelos de regressão linear 
múltipla que foram testados para explorar o peso que cada variável tinha para explicar a sintomatologia depressiva à imagem corporal.

O estudo foi controlado para as variáveis idade, sexo, escolaridade, tipo de cirurgia e tempo de follow-up cirúrgico, testando associações univariadas entre estas variáveis e a sintomatologia depressiva, que se revelaram não significativas. Tendo em conta que as variáveis independentes a incluir nos modelos de regressão devem estar significativamente correlacionadas, nas análises univariadas, com a variável dependente, estas variáveis não foram incluídas no modelo de regressão, sem prejuízo para a validade da análise.

Uma vez que o tempo de follow-up cirúrgico foi bastante alargado, e de forma a perceber se o tempo pós-cirurgia se correlacionava com variação da sintomatologia depressiva ou da imagem corporal após a cirurgia, foram realizadas algumas análises complementares. Assim sendo, dividimos a amostra em dois grupos de tempo pós-cirúrgico: os indivíduos que realizaram a cirurgia entre os 2 e os 5 anos e os que realizaram a cirurgia há mais de cinco anos. Foi testada a correlação univariada entre esta nova variável 'grupos de tempo pós-cirurgia', a sintomatologia depressiva e a imagem corporal. Os resultados demonstraram que não há associação significativa entre a variável 'grupos de tempo pós-cirurgia' e a sintomatologia depressiva $\left(r_{\mathrm{pb}}=0,12\right.$, $p=0,43)$., verificando-se o mesmo com a variável imagem corporal $\left(r_{\mathrm{pb}}=0,05, p=0,74\right)$. Ainda assim, após estas primeiras análises, a variável 'grupos de tempo pós-cirurgia' foi incluída nos modelos de regressão como covariável e os resultados demonstraram a inexistência de associação entre a variável independente 'grupos de tempo pós-cirurgia' e as variáveis dependentes sintomatologia depressiva e imagem corporal. Assim estes resultados demonstram que a sintomatologia depressiva e a imagem corporal não variam significativamente com o tempo após a cirurgia na nossa amostra. Deste modo, e uma vez que o objetivo deste estudo é analisar a variância das variáveis depressão e imagem corporal após a cirurgia, no modelo final de regressão foram incluídas variáveis, que numa primeira análise, se mostraram significativamente correlacionadas com a sintomatologia depressiva e a imagem corporal: ingestão alimentar compulsiva (IAC), a percentagem de IMC ganho (\%IMCG) após a cirurgia e a preocupação com a imagem corporal (BSQ), o comprometimento psicológico (Score Total OQ45) e a percentagem de excesso de IMC perdido (\%EIMCP).

As análises estatísticas foram realizadas em software IBM SPSS, Versão 20. A significância clinica foi considerada quando $p<0,05$. Diferenças no tamanho da amostra ao nível da perda de controlo alimentar foram devidas ao facto de não ser possível obter essas informações em quatro sujeitos.

\section{RESULTADOS}

A amostra foi constituída por 52 participantes com um período pós-cirúrgico entre os 22 e os 132 meses. Destes,
$38(73,1 \%)$ tinham realizado a banda gástrica, 9 o sleeve gástrico $(17,3 \%)$ e $5(9,6 \%)$ tinham sido submetidos ao bypass gástrico. A Tabela 1 apresenta as informações sociodemográficas e clínicas relevantes da amostra

Como se pode observar na Tabela 2, os resultados revelaram que a sintomatologia depressiva está positivamente associada à preocupação com a imagem corporal, à escala global do OQ45, à percentagem de IMCG e a episódios de IAC. Verificou-se também uma associação negativa marginalmente significativa entre a sintomatologia depressiva e a percentagem EIMCP. Nesse sentido, maior sintomatologia depressiva após a cirurgia está associada a uma maior insatisfação com a imagem corporal, a mais sintomas de comprometimento psicológico, dificuldades interpessoais e no desempenho social, a uma maior percentagem de IMCG e à presença de episódios de IAC. Adicionalmente, maior sintomatologia depressiva parece estar associada a uma menor percentagem EIMCP após a cirurgia.

De acordo com o teste de regressão linear múltipla, considerando a sintomatologia depressiva como variável dependente e a ingestão alimentar compulsiva (IAC), a $\%$ de IMC ganho (\%IMCG) após cirurgia e a preocupação com a imagem corporal (BSQ) como variáveis independentes, verificaram-se associações significativas destas variáveis com a sintomatologia depressiva. A exclusão do OQ45 nesta análise deveu-se ao facto de se pretender investigar modelos que contemplem aspetos mais específicos relacionados com o peso, a alimentação e com a imagem corporal em vez de sintomatologia geral. Os resultados mostraram que na sua totalidade este modelo de regressão linear múltipla explica $50 \%$ da variância da sintomatologia depressiva após a cirurgia $\left(R^{2}{ }_{A j}=0,46\right)$, sendo significativo $F(3,43)=$ $14,117, p<0,001$. A preocupação com a imagem corporal (BSQ) revelou-se o contributo mais significativo para a variância da sintomatologia depressiva após a cirurgia, pelo que maior insatisfação com a imagem corporal estava associada a mais sintomas depressivos, $\beta=0,535, t=3,71$, $p=0,001$ (Tabela 3).

Para melhor compreender o que estava a contribuir para a presença de sintomatologia depressiva após a cirurgia, exploraram-se as variáveis que estariam associadas à preocupação com a imagem corporal (BSQ), visto esta ter revelado um contributo bastante significativo para a presença de sintomas depressivos após a cirurgia. Foi utilizado um modelo de regressão linear múltipla, considerando como variável dependente a preocupação com a imagem corporal (BSQ) e como variáveis independentes a escala total do OQ45, a percentagem EIMCP e episódios de IAC. Este modelo explicou $56 \%$ da variância da preocupação com a imagem corporal $\left(R_{A j}^{2}=0,53\right)$, sendo significativo $(F(3,42)=18,139, p<0,001)$ e demonstrando que a escala global do OQ45 e a IAC foram as variáveis mais associadas à preocupação com a imagem corporal. Ou seja, os resultados revelaram que maior comprometimento psicológico e défices no funcionamento social, $\beta=0,460$, $t=4,11, p<0,001$, assim como a presença de episódios de IAC, $\beta=0,332, t=2,892, p=0,006$ estão associados 
Tabela 1 - Informações sociodemográficas e clínicas da amostra

\begin{tabular}{|c|c|c|c|}
\hline & \multicolumn{3}{|c|}{$n(\%)$} \\
\hline \multicolumn{4}{|l|}{ Género } \\
\hline & Feminino & \multicolumn{2}{|l|}{$43(82,7 \%)$} \\
\hline & Masculino & \multicolumn{2}{|l|}{$9(17,3 \%)$} \\
\hline \multicolumn{4}{|l|}{ Grau de obesidade } \\
\hline & Peso normal & \multicolumn{2}{|l|}{$7(13,5 \%)$} \\
\hline & Pré-obesidade & \multicolumn{2}{|l|}{$17(32,6 \%)$} \\
\hline & Obesidade grau I & \multicolumn{2}{|l|}{$7(13,5 \%)$} \\
\hline & Obesidade grau II & \multicolumn{2}{|l|}{$14(26,9 \%)$} \\
\hline & Obesidade grau III & \multicolumn{2}{|l|}{$7(13,5 \%)$} \\
\hline \multicolumn{4}{|c|}{ Aumento de peso significativo (> $5 \%$ peso corporal) } \\
\hline & Presença & \multicolumn{2}{|l|}{$31(59,6 \%)$} \\
\hline & Ausência & \multicolumn{2}{|l|}{$21(40,4 \%)$} \\
\hline \multicolumn{4}{|c|}{ Presença de episódios de IAC $(N=48)$} \\
\hline & Presença & \multicolumn{2}{|l|}{$10(19,2 \%)$} \\
\hline & Ausência & \multicolumn{2}{|l|}{$38(73,1 \%)$} \\
\hline & Min. & Max. & $M(D P)$ \\
\hline Idade & 25 & 66 & $44,04(10,87)$ \\
\hline $\begin{array}{l}\text { Tempo de Follow-Up } \\
\text { (em meses) }\end{array}$ & 22 & 132 & $53,65(37,12)$ \\
\hline Pontuações Totais de BDI & 0 & 29 & $5,25(5,67)$ \\
\hline Pontuações Totais do BSQ & 6 & 188 & $71,84(36,78)$ \\
\hline Pontuações Totais OQ45 & 7 & 78 & $42,43(17,49)$ \\
\hline$\%$ EIMCP & 0 & 90 & $16,48(21,63)$ \\
\hline$\%$ IMCG & 7,55 & 131,32 & $67,70(31,32)$ \\
\hline
\end{tabular}

Min - mínimo; Max - máximo; $M$ - média; DP - desvio padrão; IAC - Ingestão alimentar compulsiva; BDI - Beck Depression Inventory; BSQ - Body Shape Questionnaire; OQ45 Outcome Questionnaire45; \%EIMCP - Percentagem de excesso de índice de massa corporal perdido; \%IMCG - Percentagem de índice de massa corporal ganho.

Tabela 2 - Associação entre as variáveis BDI, BSQ, OQ45, \%IMCG, \%EIMCP e IAC

\begin{tabular}{|c|c|c|c|c|c|c|}
\hline & BDI & $B S Q$ & OQ45 & \%IMCG & \%EIMCP & IAC \\
\hline BDI & - & & & & & \\
\hline$B S Q$ & $0,672^{\star * *}$ & - & & & & \\
\hline OQ45 & $0,679 * * *$ & $0,648^{\star * *}$ & - & & & \\
\hline \%IMCG & $0,517^{\star * * *}$ & $0,562^{\star \star *}$ & $0,519 * * *$ & - & & \\
\hline \%EIMCP & $-0,258^{+}$ & 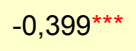 & $-0,238^{+}$ & $-0,636^{\star * *}$ & - & \\
\hline IAC & $0,473^{* *}$ & $0,578^{\star * *}$ & $0,395^{\star \star}$ & $0,478^{\star * *}$ & $-0,311^{*}$ & - \\
\hline
\end{tabular}

${ }^{+} p<0,10 ;{ }^{*} p<0,05 ;{ }^{* \star} p<0,01 ;{ }^{* * \star} p<0,001$

IAC - Ingestão alimentar compulsiva; BDI - Beck Depression Inventory; BSQ - Body Shape Questionnaire; OQ45 - Outcome Questionnaire45; \%EIMCP - Percentagem de excesso de índice de massa corporal perdido; \%IMCG - Percentagem de índice de massa corporal ganho.

a maior insatisfação com imagem corporal após a cirurgia. Adicionalmente, a percentagem EIMCP revelou-se como um contributo marginalmente significativo para a variação da preocupação com a imagem corporal após a cirurgia, sendo que uma menor percentagem EIMCP após a cirurgia parece estar associada a uma maior insatisfação com a imagem corporal $\beta=-0,192, t=-1,767, p=0,085$ (Tabela 4).

\section{DISCUSSÃO}

Os resultados deste estudo mostraram que um subgrupo de doentes apresenta um aumento de peso significativo após a cirurgia bariátrica que está associado a episódios de IAC, a preocupações com a imagem corporal e a sintomatologia depressiva. Neste sentido, a sintomatologia depressiva está mais associada a questões relacionadas com o aumento de peso do que com a perda de peso, depois da 
Tabela 3 - Modelo de Regressão Linear Múltipla considerando o BDI como variável dependente e a IAC, a \%IMCG e o BSQ como variáveis independentes

\begin{tabular}{|c|c|c|c|c|}
\hline & $R^{2}\left(R^{2}\right.$ aj) & $F(3,43)$ & $\beta$ & $t^{t}(p)$ \\
\hline IAC & & & 0,062 & $\begin{array}{l}0,46 \\
(0,652)\end{array}$ \\
\hline \%IMCG & $0,50(0,46)$ & $14,12^{\star \star \star}$ & 0,193 & $\begin{array}{l}1,41 \\
(0,166)\end{array}$ \\
\hline$B S Q$ & & & 0,535 & $\begin{array}{l}3,71^{* *} \\
(0,001)\end{array}$ \\
\hline
\end{tabular}

${ }^{* \star} p<0,01 ;{ }^{* \star \star} p<0,001$

IAC - Ingestão alimentar compulsiva; BSQ - Body Shape Questionnaire; \%IMCG - Percentagem de índice de massa corporal ganho; BDI - Beck Depression Inventory; BSQ - Body Shape Questionnaire

Tabela 4 - Modelo de Regressão Linear Múltipla considerando o BSQ como variável dependente e a escala total OQ45, IAC e \%EIMCP como variáveis independentes

\begin{tabular}{|c|c|c|c|c|}
\hline & $R^{2}\left(R^{2}\right.$ aj) & $F(3,43)$ & $\beta$ & $\begin{array}{l}t \\
(p)\end{array}$ \\
\hline Score Total OQ45 & & & 0,460 & $\begin{array}{l}4,11^{\star * \star} \\
(0,000)\end{array}$ \\
\hline IAC & $0,56(0,53)$ & $18,14^{\star \star \star}$ & 0,332 & $\begin{array}{l}2,89 * \star \\
(0,006)\end{array}$ \\
\hline \%EIMCP & & & $-0,192$ & $\begin{array}{l}-1,77^{+} \\
(0,085)\end{array}$ \\
\hline
\end{tabular}

IAC - Ingestão alimentar compulsiva; BSQ - Body Shape Questionnaire; OQ45 - Outcome Questionnaire45; \%EIMCP - Percentagem de excesso de índice de massa corporal perdido

cirurgia.

Maior sintomatologia depressiva após a cirurgia está associada a um maior comprometimento psicológico e défices no funcionamento social, à presença de episódios de IAC, a uma maior percentagem de IMC ganho e a maior insatisfação com a imagem corporal. Verificou-se que a combinação das variáveis percentagem de IMC ganho, IAC e preocupação com a imagem corporal explicam $50 \%$ da variância da sintomatologia depressiva após a cirurgia, sendo a preocupação com a imagem corporal o contributo mais significativo desta variância. Portanto, a combinação de características associadas com o comportamento alimentar, com o ganho de peso e com a imagem corporal explicam consideravelmente a variação da sintomatologia depressiva após a cirurgia.

Constatou-se ainda que maior insatisfação com a imagem corporal associa-se a uma menor percentagem de EIMCP, a presença de episódios de IAC e a um maior comprometimento psicológico e défices no funcionamento social, sendo que, em conjunto, estas variáveis explicam $56 \%$ da variação da preocupação com a imagem corporal após a cirurgia, com um contributo maior da presença de episódios de IAC e da escala global do OQ45 para esta variância. Estes resultados demonstram que a preocupação com a imagem corporal após a cirurgia é melhor explicada pela combinação de aspetos psicológicos e sociais do indivíduo e aspetos do comportamento alimentar.

Curiosamente, mesmo apesar da perda de peso inicial que todos os doentes apresentaram após cirurgia, os resultados deste estudo mostram que as variáveis associadas à sintomatologia depressiva em doentes após cirurgia bariátrica são semelhantes às variáveis associadas à depressão na população obesa que são reportadas por outros estudos. Vários estudos demonstraram que associadas à depressão na obesidade estavam variáveis como a ingestão alimentar compulsiva ${ }^{6}$, insatisfação com a imagem corporal ${ }^{8}$ e o ganho de peso ${ }^{6}$ e que pessoas com obesidade experienciavam um maior comprometimento psicológico e défices nas relações interpessoais em comparação com pessoas sem obesidade, estando estas variáveis na origem da relação entre depressão e obesidade. ${ }^{5}$

Os resultados deste estudo sugerem ainda que as variáveis que se mostram associadas à imagem corporal após a cirurgia são semelhantes às que têm vindo a ser associadas à população com obesidade, que não realizou a cirurgia. Estudos anteriores mostram associações entre a insatisfação com a imagem corporal e a presença de episódios de IAC, ${ }^{38}$ de um maior comprometimento psicológico e de défices nas relações interpessoais. ${ }^{15,16,19}$

A constatação de que as variáveis associadas à sintomatologia depressiva bem como à preocupação com a imagem corporal são semelhantes na população pós-cirurgia e na população com obesidade poderá dever-se ao facto de, apesar da significativa perda de peso inicial, na nossa amostra, mais de $50 \%$ dos indivíduos ainda apresenta níveis de pré-obesidade, obesidade I, II, ou III ${ }^{40}$ e que 31 
$(59,6 \%)$ participantes apresentam um aumento significativo do peso (> $5 \%$ do peso corporal). Além disso, o facto de a nossa amostra ter um tempo pós-cirúrgico bastante alargado poderá também justificar esta constatação.

Outro resultado interessante demonstrou que uma menor percentagem EIMCP estava associada a uma maior insatisfação com a imagem corporal após a cirurgia. A literatura tem sido pouco consistente relativamente a este aspeto, uma vez que alguns estudos mostraram que os doentes que perderam mais peso apresentavam maior insatisfação com a sua imagem corporal, devido ao surgimento de pregas cutâneas, ${ }^{11}$ e que os indivíduos que estavam mais satisfeitos com a sua imagem corporal após a cirurgia demonstravam uma menor perda de peso. ${ }^{40}$ Uma justificação para os nossos resultados prende-se com o ganho de peso verificado que está relacionado com maior insatisfação corporal.

Em suma, os resultados mostram que os indivíduos que ganharam peso após a cirurgia, apesar de uma perda ponderal inicial significativa, apresentam mais episódios de IAC, encontram-se mais insatisfeitos com a sua imagem corporal e apresentam sintomatologia depressiva mais elevada. Além disto, uma maior insatisfação com a imagem corporal apareceu também associada à presença de episódios de IAC, a maior comprometimento psicológico e défices no funcionamento social, e a uma menor percentagem de EIMC perdido ou a uma maior percentagem de IMC ganho.

Este estudo apresenta algumas limitações: o facto de seguir um design transversal não permite estabelecer relações causa-efeito quando se exploram as variáveis associadas à sintomatologia depressiva após a cirurgia, o estudo dos preditores de sintomatologia depressiva a longo prazo está limitado, sendo necessárias observações mais prospetivas e a longo-prazo de forma a compreender meIhor os efeitos da IAC, da percentagem de IMC ganho e da preocupação com a imagem corporal na sintomatologia depressiva.

Estudos futuros devem investir no uso de amostras longitudinais, no sentido de serem possíveis análises de relações causa-efeito no estudo das variáveis associadas à sintomatologia depressiva após a cirurgia, assim como no aumento do tamanho das amostras estudadas de forma a incluírem mais variáveis no estudo dos contributos para a sintomatologia depressiva após a cirurgia. É também pertinente realizar análises que possam explorar o papel mediador das variáveis associadas à preocupação com a imagem corporal na sintomatologia depressiva, uma vez que esta se revelou como um contributo bastante significativo para a variação da sintomatologia depressiva após a cirurgia. Outra questão relevante a ser explorada noutros estudos, prende-se com a hipótese de ser o ganho de peso que está associado à sintomatologia depressiva ou se é a sintomatologia depressiva que está associada ao ganho de peso, uma vez que neste estudo não foi possível estabelecer essa relação.

\section{CONCLUSÃO}

Apesar da cirurgia bariátrica ser o tratamento preferencial para obesidade grave, os resultados deste estudo enfatizam a importância de considerar a sintomatologia e, em particular, o papel das preocupações com a imagem corporal, na abordagem clínica da pessoa que fez cirurgia bariátrica de modo a otimizar o funcionamento psicológico destes doentes. As questões clínicas na abordagem de uma condição crónica como a Obesidade não devem ser descuradas no acompanhamento pós-cirúrgico que deverá ser considerado ao longo da vida do paciente, de modo a prevenir o aumento de peso e, eventualmente, piores resultados do tratamento.

\section{AGRADECIMENTOS}

Os autores gostariam de agradecer a António Sérgio Bastos Silva (Clinica Dr. António Sérgio, Unidade de Bariatria do Instituto CUF Porto) pelo envolvimento e apoio na recolha de dados.

\section{CONFLITOS DE INTERESSE}

Os autores declaram a inexistência de conflitos de interesse.

\section{FONTES DE FINANCIAMENTO}

Esta investigação foi parcialmente suportada pela Fundação para a Ciência e Tecnologia, Portugal através do projeto (PTDC/MHC-PCL/4974/2012) e bolsa (SFRH/ BPD/78896/2011) concedidos a Eva Conceição, e do projeto (PTDC/PSI-PCL/099981/2008) concedido a Paulo Machado.

\section{REFERÊNCIAS}

1. Beck NN, Johannsen M, Støving RK, Mehlsen M, Zachariae R. Do postoperative psychotherapeutic interventions and support groups influence weight loss following bariatric surgery? A systematic review and meta-analysis of randomized and nonrandomized trials. Obes Surg. 2012;22:1790-7

2. Libeton M, Dixon JB, Laurie C, O'Brien PE. Patient motivation for bariatric surgery: characteristics and impact on outcomes. Obes Surg. 2004;14:392-8.

3. Zuegel NP, Lang RA, Hüttl TP, Gleis M, Ketfi-Jungen M, Rasquin I, Kox $M$. Complications and outcome after laparoscopic bariatric surgery: LAGB versus LRYGB. Langenbecks Arch Surg. 2012;397:1235-41.

4. do Carmo I, Dos Santos O, Camolas J, Vieira J, Carreira M, Medina L, et al. Overweight and obesity in Portugal: national prevalence in 2003-

2005. Obes Rev. 2008;9:11-9.

5. Luppino FS, de Wit LM, Bouvy PF, Stijnen T, Cuijpers P, Penninx BW, et al. Overweight, obesity, and depression: a systematic review and metaanalysis of longitudinal studies. Arch Gen Psychiatry. 2010;67:220-9.

6. Bulik CM, Sullivan PF, Kendler KS. An empirical study of the classification of eating disorders. JAMA Psychiatry. 2000;157:886-95.

7. Werrij MQ, Mulkens S, Hospers HJ, Jansen A. Overweight and obesity: the significance of a depressed mood. Patient Educ Couns. 2006;62:126-31.

8. Rosenberger PH, Henderson KE, Grilo CM. Correlates of body image dissatisfaction in extremely obese female bariatric surgery candidates. Obes Surg. 2006;16:1331-6.

9. van Hout G. Psychosocial effects of bariatric surgery. Acta Chir Belg. 
2005;105;40-3.

10. Sarwer DB, Thompson JK, Cash TF. Body image and obesity in adulthood. Psychiatr Clin North Am. 2005;28:69-87.

11. Dixon JB, Dixon ME, O’Brien PE. Body image: appearance orientation and evaluation in the severely obese: changes with weight loss. Obes Surg. 2002;12:65-71.

12. Cooper Z, Fairburn CG. A new cognitive behavioral approach to the treatment of obesity. Behav Res Ther. 2001;39:499-511.

13. Hsu LK, Benotti PN, Dwyer J, Roberts SB, Saltzman E, Shikora S, et al. Nonsurgical factors that influence the outcome of bariatric surgery: a review. Psychosom Med. 1998;60:338-46.

14. Averbukh Y, Heshka S, El-Shoreya H, Flancbaum L, Geliebter A, Kamel $S$, et al. Depression score predicts weight loss following Roux-en-Y gastric bypass. Obes Surg. 2003;13:833-6.

15. Bocchieri LE, Meana M, Fisher BL. A review of psychosocial outcomes of surgery for morbid obesity. J Psychosom Res. 2002;52:155-65.

16. Sarwer DB, Wadden TA, Fabricatore AN. Psychosocial and behavioral aspects of bariatric surgery. Obes Res. 2005;13:639-48.

17. Buchwald $H$, Avidor $Y$, Braunwald $E$, Jensen MD, Pories W, Fahrbach $\mathrm{K}$, et al. Bariatric Surgery: a systematic review and meta-analysis. J Am Med Assoc. 2004;292:1724-8.

18. Mitchell JE, Crosby R, De Zwaan M, Engel S, Roerig J, Steffen K, et al. Possible risk factors for increased suicide following bariatric surgery. Obesity. 2012;21:665-72.

19. van Hout G, Verschure SKM, van Heck GL. Psychosocial predictors of success following bariatric surgery. Obes Surg. 2005;15:552-60.

20. Greenberg I, Perna F, Kaplan M, Sullivan MA. Behavioral and psychological factors in the assessment and treatment of obesity surgery patients. Obes Res. 2005;13:244-9.

21. van Hout G, Hagendoren CA, Verschure SK, van Heck GL. Psychosocial predictors of success after vertical banded gastroplasty. Obes Surg. 2009:701-7.

22. World Health Organization. Who.int. Geneva: Obesity and overweight; [consultado 2013 Mar 10]. Disponível em http://www.who.int/mediacentre/factsheets/fs311/en/.

23. Legenbauer T, Herpertz S, de Zwaan M. Psychosocial problems and psychiatric disorders pre- and postbariatric surgery. In: Mitchell JE, de Zwaan M, editors. Psychosocial assessment and treatment of bariatric surgery patients. New York: Routledge; 2012. p. 61-85.

24. de Zwaan M, Enderle J, Wagner S, Mühlhans B, Ditzen B, Gefeller O et al. Anxiety and depression in bariatric surgery patients: a prospective, follow-up study using structured clinical interviews. J Affect Disord. 2011;133:61-8

25. Niego SH, Kofman MD, Weiss JJ, Geliebter A. Binge eating in the bariatric surgery population: a review of the literature. Int $\mathrm{J}$ Eat Disord. 2007;40:349-59.
26. Sjöström L. Review of the key results from the Swedish Obese Subjects (SOS) trial - a prospective controlled intervention study of bariatric surgery. J Intern Med. 2013;273:219-34.

27. White MA, Kalarchian MA, Masheb RM, Marsha D, Grilo CM. Loss of control over eating predicts outcomes in bariatric surgery: a prospective 24- month follow-up study. J Clin Psychiatry. 2011;71:175-84.

28. Fairburn CG, Cooper Z. The eating disorder examination. In: Fairburn CG, Wilson GT, editors. Binge eating: nature, assessment and treatment. New York: Guilford Press; 1993. p. 317-60.

29. Machado PP, Machado BC, Gonçalves S, Hoek HW. The prevalence of eating disorders not otherwise specified. Int J Eat Disord. 2007;40:2127.

30. Wilfley DE, Schwartz MB, Spurrell EB, Fairburn CG. Using the eating disorder examination to identify the specific psychopathology of binge eating disorder. Int J Eat Disord. 2000;27:259-69.

31. Cooper Z, Cooper PJ, Fairburn CG. The validity of the eating disorder examination and its subscales. Br J Psychiatry. 1989;154:807-12.

32. Vaz Serra A, Pio Abreu JL. Aferição dos quadros clínicos depressivos: I - Ensaio de aplicação do inventário depressivo de Beck a uma amostra portuguesa de doentes deprimidos. Coimbra Med. 1973;20:623-44.

33. Vaz Serra A, Pio Abreu JL. Aferição dos quadros clínicos depressivos: II - Estudo preliminar de novos agrupamentos sintomatológicos para complemento do inventário depressivo de Beck. Coimbra Med. 1973;20:713-36.

34. Beck A, Steer R, Garbin M. Psychometric properties of the Beck Depression Inventory: twenty-five years of evaluation. Clin Psychol Rev. 1988;8:77-100.

35. Lambert MJ, Burlingame GM, Umphress V, Hansen NB, Vermeersch DA, Clouse GC, et al. The reliability and validity of the Outcome Questionnaire. Clin Psychol Psychother. 1996;3:249-58.

36. Cooper PJ, Taylor MJ, Cooper Z, Fairbun CG. The development and validation of the body shape questionnaire. Int J Eat Disord. 1987;6:48549

37. Vieira PN, Palmeira, AL, Francisco C, Teixeira PJ. Validação da versão portuguesa do questionário da forma corporal. $5^{\circ}$ Congresso da Psicologia da Saúde. 2004.

38. Grilo CM, Masheb RM, Brody M, Burke-Martindale $\mathrm{CH}$, Rothschild BS. Binge eating and self-esteem predict body image dissatisfaction among obese men and women seeking bariatric surgery. Int J Eat Disord. 2005;3:347-51.

39. World Health Organization. Who.int. Geneva: Global Database on Body Mass Index; [consultado 2013 Abr 11]. Disponível em http://apps.who. int/bmi/index.jsp.

40. van Hout GC, Boekestein P, Fortuin FA, Pelle AJ, van Heck GL. Psychosocial functioning following bariatric surgery. Obes Surg. 2006;16;78794 

MACHADO, Eva CONCEIÇÃO

\section{Compreender a Sintomatologia Depressiva após a Cirurgia Bariátrica: o Papel do Peso, da Alimentação e da Imagem Corporal Acta Med Port 2014:27:450-457}

Publicado pela Acta Médica Portuguesa, a Revista Científica da Ordem dos Médicos

Av. Almirante Gago Coutinho, 151

1749-084 Lisboa, Portugal.

Tel: +351 218428215

E-mail: submissao@actamedicaportuguesa.com

www.actamedicaportuguesa.com

ISSN:0870-399X | e-ISSN: 1646-0758

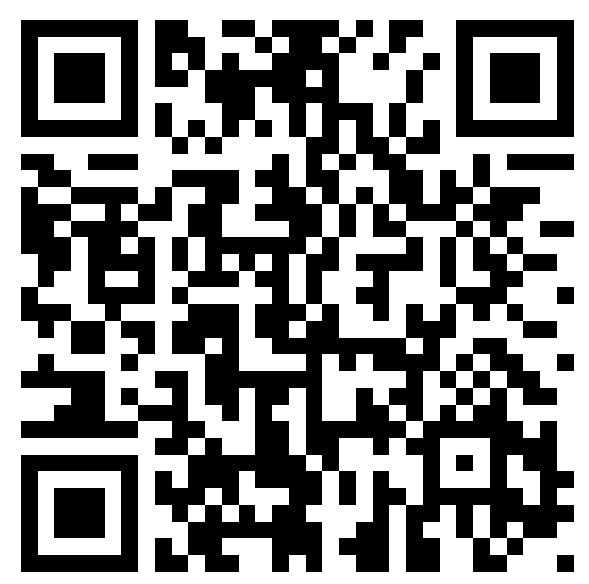

